\title{
Impact of Mikania micrantha Kunth ex HBK Invasion on Micro-environment Component of Bherjan- Borajan-Podumoni Wild Life Sanctuary, Assam
}

\author{
Kuntala Neog Barua*, Indrani P. Bora and Arundhati Baruah
}

Rain Forest Research Institute, Sotai, Jorhat, India

*Corresponding author

\section{Keywords}

Microenvironment component, Impact, Mikania micrantha invasion, Bherjan-

Borajan-Podumoni WLS, Assam

Article Info

Accepted:

20 August 2020

Available Online:

10 September 2020

\section{A B S T R A C T}

\section{Introduction}

The stability and ecological function of natural wild lands depend on a diverse population of native plants. Native vegetation provided resilience against drought and flood, minimizes erosion, and promotes water infiltration and storage, along with providing wildlife and recreation values. Otherwise, the forest community was altered due to habitat 
fragmentation or invasion of foreign species (Pimm, 1986). Any biological species which establishes outside its native habitat and aggressively out-competes the native species was called an invasive alien species (Tiwari, 2005). Convention on Biological Diversity (1992) emphasized on the invasion of alien species which was considered as the second worst threat for biodiversity.

The invasive plants differ from native one, on their requirements and more consumption that may cause changes in soil structure, profile, decomposition rate, moisture and nutrient content, Singh (2001). Drake et al., (1989), Brown et al., (1998) also stated that exotic invasion in the natural ecosystem have undesirable impacts on economic and ecological sustainability. Wide-ranging infestations of weeds can permanently degrade natural forest ecosystem. Ehrenfeld (2003) declared that changes of vegetation composition due to exotic invasion are always connected with the changes of soil properties.

Mikania micrantha Kunth. ex. H.B.K. is one of the world's most aggressive tropical alien perennial vines of Asteraceae family native from South and Central America and placed amid the 10 worst exotic species in South and South East Asia (Lowe et al., 2000).

Because of its vigorous and ramet growth habit, it successfully competes with trees and other crop plants for soil nutrients, water and reduces sunlight interception by covering the canopy. The open patches of the forest of upper Assam severely infested by this weed and might be the prime factor for forest conversion.

Therefore, the present study was undertaken to evaluate the changes of microenvironment component of Bherjan- Borajan - Podumoni Wild Life Sanctuary, Assam due to infestation of M. micrantha.

\section{Materials and Methods}

\section{Study site}

Bherjan, Borajan and Podumoni were the three tiny pockets of tropical forest on the flat plains of the Brahmputra Valley named Bherjan-Borjan-Padumoni Wild Life Sanctuary (WLS) situated in Tinsukia district of Assam in between the coordinates of $27^{\circ} 25^{\prime} 22.9^{\prime \prime} \mathrm{N}$ to $27^{\circ} 32$ ' $24^{\prime \prime} \mathrm{N}$ and 95 $18^{\prime} 40.5^{\prime \prime} \mathrm{E}$ to $95^{\circ} 29^{\prime} 16.4^{\prime \prime} \mathrm{E}$ (Figure 1). This was a small WLS which covered an area of 7.22 sq.km with altitude range of $119-122$ $\mathrm{m}$ from MSL. The three small forest segments were disjuncted by tea gardens and human habitations. The natural vegetation is Tropical Wet Evergreen 'rainforest' type experiences a subtropical humid monsoon climate with an average annual rainfall of $2263 \mathrm{~mm}$. Average maximum temperature varied from $24^{\circ} \mathrm{C}$ to $33.9^{\circ} \mathrm{C}$ and minimum temperature from $6.9^{\circ} \mathrm{C}$ to $24.6^{\circ} \mathrm{C}$. The relative humidity was highest at $88 \%$ in the month of June and January was the driest month having average relative humidity was $70.6 \%$. Soil was rich loamy with old alluvium deposition of Brahmaputra and acidic in nature with $\mathrm{pH}$ ranging from 4.07 to 4.7 .

\section{Methodology}

Extensive field surveys were conducted during the period 2014-2018 for evaluation of impact of $M$. micrantha on microenvironment of native species in Bherjan-Borjan-Padumoni WLS. The forests were divided into two position i) $M$. micrantha un-infested, ii) $M$. micrantha infested site.

To study the micro environmental characters, the parameters like soil nutrient status, soil temperature, light interception and biomass production were taken. Soil samples were collected from the forest with three different depths of $0-15 \mathrm{~cm}, 15-25 \mathrm{~cm}$ and $25-50 \mathrm{~cm}$. 
Bulk density, $\mathrm{pH}$ and NPK were determined by the methods as described by Jackson (1973). Organic carbon percentage was estimated following modified Walkley and Black's wet oxidation method (Jha, 2005). Soil temperature was recorded through soil thermometer at seasonal intervals. Biomass study was done seasonally from $M$. micrantha infested forest sites by harvest method with twenty five replications in sample plot of $1 \mathrm{~m}$ $\mathrm{x} 1 \mathrm{~m}$. Samples were kept in the oven at 60 $70^{\circ} \mathrm{C}$ until constant weight will obtained. Total production was estimated by adding the biomass accumulation and standing dead material i.e. litter at seasonal intervals, by following Swamy and Ramakrishnan (1987 b). Population study of $M$. micrantha was carried out by randomly laid 125 quadrats of $1 \mathrm{~m} \times 1 \mathrm{~m}$ in each site. Quantitative analysis for density and Importance Value were calculated following Misra (1968) and Kershew (1973). Light intensity was recorded periodically from above the canopy and at ground level through lux meter and interception of light by the canopies was calculated by deduction method.

Correlation of environmental data, soil data with the vegetation data recorded from infested forest sites was analyzed by using SPSS 16.0 software.

\section{Results and Discussion}

Rapid invasion of $M$. micrantha in natural ecosystems during recent years was correlated with the extinction of native species (Leeand Klasing, 2004). Vigorous vegetative and sexual reproductive ability of this invader species and its population dynamics was already described by Swamy and Ramakrishnan (1987a; 1987b) for north east India. It has now become a most troublesome in the Bherjan -Borjan-Padumonbi WLS of Assam.The forest was a mosaic of disturbed open canopy patches along with little closed canopy areas. Seasonal structural changes of M. micrantha showed that the weed was strongly invaded in the forest and the maximum dominancy was recorded in the monsoon and post monsoon season. Population density of Mikania declined in pre monsoon season and minimum density was found in the month of March to April (Figure 2). Puzari (2010) reported that the population of Mikania was positively correlated with temperature. Our present study revealed a similar observation in density and biomass production of Mikania correlated to atmospheric temperature. The average maximum temperature varied from $24^{\circ} \mathrm{C}$ to $33.9^{\circ} \mathrm{C}$ in the study sites which supported the luxuriant growth of the weed. The highest infestation was recorded in Padumoni segment of the WLS with IVI of 158.90. Aravind et al., 2010 reported that in Karnataka invasion of Lantana camara found higher in open forest areas. Study also found that the most preferred habitat for growth and development of Mikania was also in open sunny place with moist soil and cannot normally tolerate most impenetrable sites. Presently it was observed that the weed flourished mostly in canopy gap areas and the peripheral region of natural forests and absent in closed shaded areas. Frequent tree felling and lopping of trees created localized canopy gap patches in the WLS and acted as window for successful invasion of Mikania in the forest.

Invasive weeds have tremendous potential to produce massive amount of biomass and Anonymous, 2007 reported that Parthenium produces $5-20 \mathrm{~kg} / \mathrm{m}^{2}, \quad 9.3 \mathrm{~kg} / \mathrm{m}^{2}$ in Chromolaena, $3 \mathrm{~kg} / \mathrm{m}^{2}$ in Cassia, $1-1.5 \mathrm{~kg} / \mathrm{m}^{2}$ in Lantana and $10-11.5 \mathrm{~kg} / \mathrm{m}^{2}$ in Water hyacinth. Mikania also contributed a notable amount of biomass in the studied infested forest areas. Biomass accumulation was high in post monsoon season for all the forest sites and it was found maximum in Padumoni 
segment (2603.3 $\mathrm{kg} / \mathrm{ha})$ during the full growing season. Seasonal Biomass and Litter Production ( $\mathrm{kg} / \mathrm{ha}$ ) of Mikania and other species was given in Table 1 .

Average maximum soil temperature was observed in monsoon season of infested forest site of all three segments. The summer temperature of WLS was varied from $30.23^{\circ} \mathrm{C}$ \pm 0.50 to $32.37^{\circ} \mathrm{C} \pm 0.55$ and Padumoni segment exhibited the highest one. In uninfested natural forest site the temperature goes up to $31.3^{0} \mathrm{C} \pm 0.56$ in monsoon season (Table 2).

The exotic invader has a greater capacity to efficiently utilize light energy by producing more foliage that essential for growth and development (Feng et al., 2007).Comparative assessment of light interception percentage and Mikania biomass at different growth season revealed that the highest biomass was accumulated in the post monsoon season related to maximum light intercepted by the Mikania canopy. In pre monsoon season Mikania seedlings were in sprouting condition hence, intercepted minimum extent of light (Figure 3).

In forest ecosystems the understory plants were highly sensitive to their micro environment governed by the light intensity, wind velocity, temperature, soil moisture, etc. Any change of these environmental parameters might be detrimental for the existence of some of the sensitive species as seen due to invasion of Mimosa diplotrica, in Kaziranga National Park of Assam or $M$. micrantha in Chitwan National Park of Nepal (Vasu, 2003; Ram, 2008). Infestation of $M$. micrantha was considered as of high risk as it smothered the vegetation causing tree death. Presence of the weed in the canopy gaps where light intensity is higher which indicated that an increase in light through disturbance in the forest canopy encourages invasion into the forest. The dense shade below canopies of the weed suppresses the growth of other plant species (Huang et al., 2000). Mikania caught up light intensity on ground surface of the forest and affected the regeneration of several tree seedlings. Invasion of the weed supported the enrollment of some light demanding deciduous species such as Alstonia scholaris, Balakata baccata, Bombax ceiba, Ficus hispida, Lagerstroemia speciosa, Premna latifolia, which facilitated the expansion of the weed and helped in degradation of the forest. Once the weed colonized the areas they increasingly penetrated the forest very tactfully. Through rapid proliferation and ramet formation, the weed entangled immediately the crown of the sapling and small seedlings. Sankaran et al., (2001) was reported similar problem in case of young plantation of Teak in Kerala. Barua and Hazarika (2016) also confirmed that Mikania encourage the alteration of evergreen species in Dilli reserve forest of Assam.

Physico chemical properties of soil in different seasons in both infested and uninfested sites of Bherjan-Borjan-Padumoni WLS were given in Table 3-5. pH value ranges from 4.07 to 4.70 which indicate acidic nature of soil, this may be due to release of acidic compounds by the decomposition of organic residues from forest vegetation (Gogoi et al., 2011; Acharya and Shrestha, 2012 and Anup et al., 2013). The humification and leaching of basic ions were the two contributing factors along with rainfall and acidic parent materials. Comparatively high amount of litterfall in un infested natural forest contributed more organic matter to soil ultimately creating favourable environment to microbes responsible for production of organic acids into soil (Jha et al., 1979).

Moisture Content of the soil was changeable according to the quantity of rainfall in the 
study sites. It was recorded more in uninfested forest site than the infested site and gradually increased with increasing depth. Maximum proliferation of surface absorbing roots of $M$. micrantha contributed to higher absorption of moisture in the surface layer. Highest moisture content was recorded in natural forest site of Bherjan segment (39.87 \pm 0.03 due to high humid closed canopy cover of Dipterocarp-Mesua-Artocarpus association. The percentage of moisture was higher in monsoon and post monsoon season (Table 3).

Table.1 Biomass and Litter Production (kg/ha) in Infested sites of Bherjan-Borjan-Padumoni WLS

\begin{tabular}{|l|l|l|l|l|}
\hline Season & Mikania & Other & Litter & Total Production \\
\hline Pre-monsoon & $428.6 \pm 0.39$ & $320 \pm 0.07$ & $909.3 \pm 0.72$ & $1657.9 \pm 0.99$ \\
\hline Monsoon & $960.3 \pm 0.48$ & $728 \pm 0.28$ & $183 \pm 0.20$ & $1871.3 \pm 0.59$ \\
\hline Post-monsoon & $1103.6 \pm 0.53$ & $776.6 \pm 0.10$ & $170.6 \pm 0.29$ & $2050.8 \pm 0.37$ \\
\hline Winter & $792.6 \pm 0.20$ & $506.6 \pm 0.05$ & $846 \pm 0.04$ & $2145.2 \pm 0.25$ \\
\hline \multicolumn{5}{|c|}{ Borjan } \\
\hline Pre-monsoon & $387.6 \pm 0.24$ & $303.6 \pm 0.08$ & $684.6 \pm 0.35$ & $1375.8 \pm 0.51$ \\
\hline Monsoon & $763.6 \pm 0.17$ & $624 \pm 0.22$ & $214.3 \pm 0.20$ & $1601.9 \pm 0.22$ \\
\hline Post-monsoon & $964.6 \pm 0.36$ & $711.3 \pm 0.53$ & $264.3 \pm 0.07$ & $1940.2 \pm 0.80$ \\
\hline Winter & $455.3 \pm 0.09$ & $345 \pm 0.76$ & $805 \pm 0.34$ & $1605.3 \pm 0.99$ \\
\hline \multicolumn{5}{|c|}{ Padumoni } \\
\hline Pre-monsoon & $1154.6 \pm 0.52$ & $452.3 \pm 0.32$ & $653.6 \pm 0.11$ & $2260.50 \pm 0.24$ \\
\hline Monsoon & $2304.3 \pm 0.28$ & $596 \pm 0.93$ & $196.3 \pm 0.35$ & $3096.6 \pm 0.89$ \\
\hline Post-monsoon & $\mathbf{2 6 0 3 . 3} \pm 7.45$ & $578.6 \pm 0.31$ & $248.6 \pm 0.13$ & $3430.50 \pm 7.28$ \\
\hline Winter & $1649.3 \pm 0.16$ & $550.3 \pm 0.32$ & $404 \pm 0.59$ & $2603.6 \pm 0.64$ \\
\hline
\end{tabular}

Table.2 Seasonal variation of Light interception (\%) and Soil Temperature of Bherjan-Borjan-Padumoni WLS

\begin{tabular}{|c|c|c|c|c|c|c|}
\hline Trait & & Site & Pre Monsoon & Monsoon & Post Monsoon & Winter \\
\hline \multirow{2}{*}{$\begin{array}{l}\text { Soil } \\
\text { temperature }\left({ }^{0} \mathrm{C}\right)\end{array}$} & \multirow{4}{*}{ 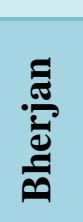 } & I & $22.40 \pm 1.05$ & $31.37 \pm 0.64$ & $27.50 \pm 1.57$ & $17.50 \pm 0.79$ \\
\hline & & UI & $21.57 \pm 1.65$ & $29.7 \pm 0.72$ & $26.00 \pm 0.92$ & $17.07 \pm 0.65$ \\
\hline \multirow{2}{*}{$\begin{array}{l}\text { Light interception } \\
(\%)\end{array}$} & & I & $45.2 \pm 0.30$ & $70.36 \pm 0.57$ & $79.75 \pm 0.06$ & $53.25 \pm 0.28$ \\
\hline & & UI & $81.33 \pm 0.40$ & $87.8 \pm 0.70$ & $87.8 \pm 0.90$ & $79.67 \pm 0.75$ \\
\hline \multirow{2}{*}{$\begin{array}{l}\text { Soil } \\
\text { temperature }\left({ }^{0} \mathrm{C}\right)\end{array}$} & \multirow{4}{*}{ 苛 } & I & $19.73 \pm 0.50$ & $30.23 \pm 0.50$ & $26.93 \pm 1.46$ & $17.53 \pm 0.75$ \\
\hline & & UI & $19.53 \pm 1.21$ & $28.17 \pm 1.15$ & $26.37^{0} \mathrm{C} \pm 0.80$ & $16.47 \pm 0.57$ \\
\hline \multirow{2}{*}{$\begin{array}{l}\text { Light interception } \\
(\%)\end{array}$} & & I & $41.82 \pm 0.76$ & $60.06 \pm 0.47$ & $65.33 \pm 0.50$ & $49.34 \pm 0.41$ \\
\hline & & UI & $82.03 \pm 1.43$ & $90.83 \pm 0.93$ & $88.4 \pm 0.62$ & $80.73 \pm 1.00$ \\
\hline \multirow{2}{*}{$\begin{array}{l}\text { Soil } \\
\text { temperature }\left({ }^{0} \mathrm{C}\right)\end{array}$} & \multirow{4}{*}{$\begin{array}{l}\ddot{\Xi} \\
\text { 音 } \\
\text { E. }\end{array}$} & I & $24.33 \pm 1.16$ & $32.37 \pm 0.55$ & $28.30 \pm 0.95$ & $19.57 \pm 1.26$ \\
\hline & & UI & $22.87 \pm 0.93$ & $31.3 \pm 0.56$ & $27.63 \pm 0.25$ & $18.17 \pm 0.81$ \\
\hline \multirow{2}{*}{$\begin{array}{l}\text { Light interception } \\
(\%)\end{array}$} & & I & $69.37 \pm 0.49$ & $86.53 \pm 0.59$ & $98.75 \pm 0.03$ & $86.25 \pm 0.52$ \\
\hline & & UI & $51.47 \pm 1.04$ & $74.27 \pm 1.11$ & $68.33 \pm 0.75$ & $61.40 \pm 1.15$ \\
\hline
\end{tabular}

I: Infested UI: Un-infested 
Table.3 pH and moisture content of soil in Bherjan-Borjan-Padumoni WLS

\begin{tabular}{|c|c|c|c|c|c|c|c|c|c|c|c|c|c|c|c|c|c|}
\hline \multirow[t]{3}{*}{ Forest } & & \multicolumn{8}{|c|}{ pH } & \multicolumn{8}{|c|}{ MOISTURE CONTENT } \\
\hline & & \multicolumn{2}{|c|}{ Pre-M } & \multicolumn{2}{|c|}{ Monsoon } & \multicolumn{2}{|c|}{ Post- M } & \multicolumn{2}{|c|}{ Winter } & \multicolumn{2}{|c|}{ Pre-M } & \multicolumn{2}{|c|}{ Monsoon } & \multicolumn{2}{|c|}{ Post- M } & \multicolumn{2}{|l|}{ Winter } \\
\hline & & IN & UI & IN & UI & IN & UI & IN & UI & IN & UI & IN & UI & IN & UI & IN & UI \\
\hline \multirow{6}{*}{$\begin{array}{l}\text { Bherjan } \\
\text { Segment }\end{array}$} & A & 4.14 & 4.11 & 4.26 & 4.17 & 4.70 & 4.32 & 4.21 & 4.12 & 16.44 & 18.20 & 26.52 & 31.28 & 20.55 & 26.85 & 22.88 & 25.27 \\
\hline & & \pm 0.02 & \pm 0.03 & \pm 0.02 & \pm 0.02 & \pm 0.02 & \pm 0.02 & \pm 0.02 & \pm 0.02 & \pm 0.03 & \pm 0.25 & \pm 0.016 & \pm 0.02 & \pm 0.02 & \pm 0.02 & \pm 0.016 & \pm 0.008 \\
\hline & B & 4.18 & 4.21 & 4.29 & 4.23 & 4.61 & 4.26 & 4.17 & 4.07 & 25.94 & 26.74 & 30.14 & 36.57 & 22.59 & 28.72 & 24.59 & 25.66 \\
\hline & & \pm 0.01 & \pm 0.02 & \pm 0.02 & \pm 0.03 & \pm 0.02 & \pm 0.01 & \pm 0.02 & \pm 0.02 & \pm 0.05 & \pm 0.05 & \pm 0.03 & \pm 0.02 & \pm 0.02 & \pm 0.02 & \pm 0.03 & \pm 0.01 \\
\hline & $\mathrm{C}$ & 4.15 & 4.15 & 4.21 & 4.19 & 4.55 & 4.22 & 4.21 & 4.13 & 28.70 & 35.86 & 32.24 & 39.87 & 25.78 & 32.82 & 26.37 & 28.26 \\
\hline & & \pm 0.01 & \pm 0.01 & \pm 0.02 & \pm 0.02 & \pm 0.03 & \pm 0.01 & \pm 0.02 & \pm 0.01 & \pm 0.04 & \pm 0.02 & \pm 0.03 & \pm 0.03 & \pm 0.01 & \pm 0.02 & \pm 0.01 & \pm 0.01 \\
\hline \multirow{6}{*}{$\begin{array}{l}\text { Borjan } \\
\text { Segment }\end{array}$} & A & 4.37 & 4.28 & 4.32 & 4.28 & 4.41 & 4.30 & 4.22 & 4.18 & 14.55 & 15.81 & 24.75 & 29.46 & 16.22 & 27.66 & 22.41 & 24.37 \\
\hline & & \pm 0.02 & \pm 0.01 & \pm 0.02 & \pm 0.02 & \pm 0.04 & \pm 0.02 & \pm 0.02 & \pm 0.03 & \pm 0.03 & \pm 0.02 & \pm 0.03 & \pm 0.04 & \pm 0.05 & \pm 0.03 & \pm 0.01 & \pm 0.03 \\
\hline & B & 4.32 & 4.24 & 4.28 & 4.14 & 4.30 & 4.26 & 4.22 & 4.15 & 16.55 & 20.62 & 28.68 & 32.56 & 21.45 & 28.74 & 26.39 & 26.88 \\
\hline & & \pm 0.02 & \pm 0.02 & \pm 0.02 & \pm 0.02 & \pm 0.04 & \pm 0.02 & \pm 0.03 & \pm 0.03 & \pm 0.02 & \pm 0.03 & \pm 0.02 & \pm 0.04 & \pm 0.02 & \pm 0.04 & \pm 0.03 & \pm 0.04 \\
\hline & $\mathrm{C}$ & 4.30 & 4.19 & 4.24 & 4.19 & 4.26 & 4.13 & 4.18 & 4.12 & 20.62 & 31.57 & 30.76 & 34.55 & 21.86 & 30.54 & 28.88 & 29.76 \\
\hline & & \pm 0.04 & \pm 0.06 & \pm 0.02 & \pm 0.02 & \pm 0.02 & \pm 0.02 & \pm 0.03 & \pm 0.04 & \pm 0.02 & \pm 0.04 & \pm 0.03 & \pm 0.03 & \pm 0.04 & \pm 0.04 & \pm 0.04 & \pm 0.03 \\
\hline \multirow{6}{*}{$\begin{array}{l}\text { Padumoni } \\
\text { Segment }\end{array}$} & A & 4.24 & 4.12 & 4.30 & 4.22 & 4.28 & 4.22 & 4.18 & 4.17 & 12.99 & 21.31 & 24.57 & 30.48 & 22.56 & 25.76 & 12.87 & 17.76 \\
\hline & & \pm 0.02 & \pm 0.05 & \pm 0.17 & \pm 0.04 & \pm 0.03 & \pm 0.04 & \pm 0.03 & \pm 0.03 & \pm 0.06 & \pm 0.03 & \pm 0.03 & \pm 0.02 & \pm 0.03 & \pm 0.05 & \pm 0.04 & \pm 0.02 \\
\hline & B & 4.22 & 4.11 & 4.27 & 4.20 & 4.25 & 4.16 & 4.11 & 4.07 & 18.76 & 22.24 & 29.42 & 34.58 & 24.59 & 25.77 & 13.55 & 18.88 \\
\hline & & \pm 0.02 & \pm 0.03 & 0.04 & \pm 0.03 & \pm 0.02 & \pm 0.02 & \pm 0.03 & \pm 0.02 & \pm 0.04 & \pm 0.04 & \pm 0.03 & \pm 0.02 & \pm 0.03 & \pm 0.03 & \pm 0.03 & \pm 0.03 \\
\hline & $\mathrm{C}$ & 4.19 & 4.05 & 4.25 & 4.19 & 4.19 & 4.12 & 4.07 & 4.08 & 19.61 & 29.87 & 33.48 & 38.46 & 26.48 & 28.84 & 19.87 & 21.84 \\
\hline & & \pm 0.05 & \pm 0.03 & 0.02 & \pm 0.02 & \pm 0.03 & \pm 0.03 & \pm 0.04 & \pm 0.03 & \pm 0.02 & \pm 0.02 & \pm 0.03 & \pm 0.04 & \pm 0.05 & \pm 0.04 & \pm 0.05 & \pm 0.05 \\
\hline
\end{tabular}

$\mathrm{I}=$ infested, $\mathrm{UI}=$ un- infested, A: $0-15 \mathrm{~cm}, \quad B: 5-25 \mathrm{~cm}, \mathrm{C}: 25-50 \mathrm{~cm}$ 
Table.4 Organic carbon and Available Nitrogen content of soil in Bherjan-Borjan-Padumoni WLS

\begin{tabular}{|c|c|c|c|c|c|c|c|c|c|c|c|c|c|c|c|c|c|}
\hline \multirow[t]{3}{*}{ Forest } & & \multicolumn{8}{|c|}{ Organic Carbon (\%) } & \multicolumn{8}{|c|}{ Available Nitrogen $\left(\mathrm{Kg} \mathrm{ha}^{-1}\right)$} \\
\hline & & \multicolumn{2}{|l|}{ Pre-M } & \multicolumn{2}{|c|}{ Monsoon } & \multicolumn{2}{|c|}{ Post- M } & \multicolumn{2}{|l|}{ Winter } & \multicolumn{2}{|l|}{ Pre-M } & \multicolumn{2}{|c|}{ Monsoon } & \multicolumn{2}{|c|}{ Post- M } & \multicolumn{2}{|c|}{ Winter } \\
\hline & & IN & UI & IN & UI & IN & UI & IN & UI & IN & UI & IN & UI & IN & UI & IN & UI \\
\hline \multirow[t]{3}{*}{$\begin{array}{l}\text { Bherjan } \\
\text { Segment }\end{array}$} & A & $\begin{array}{l}1.56 \\
\pm 0.02\end{array}$ & $\begin{array}{l}1.83 \\
\pm 0.03\end{array}$ & $\begin{array}{l}1.45 \\
\pm 0.02\end{array}$ & $\begin{array}{l}1.65 \\
\pm 0.04\end{array}$ & $\begin{array}{l}1.95 \\
\pm 0.02\end{array}$ & $\begin{array}{l}2.71 \\
\pm 0.02\end{array}$ & $\begin{array}{l}1.26 \\
\pm 0.01\end{array}$ & $\begin{array}{l}1.58 \\
\pm 0.02\end{array}$ & $\begin{array}{l}147.39 \\
\pm 0.47\end{array}$ & $\begin{array}{l}232.06 \\
\pm 0.06\end{array}$ & $\begin{array}{l}123.53 \\
\pm 0.03\end{array}$ & $\begin{array}{l}225.5 \\
\pm 0.24\end{array}$ & $\begin{array}{l}257.5 \\
\pm 0.65\end{array}$ & $\begin{array}{l}285.3 \\
\pm 0.57\end{array}$ & $\begin{array}{l}107.6 \\
\pm 0.28\end{array}$ & $\begin{array}{l}98.5 \\
\pm 0.44\end{array}$ \\
\hline & B & $\begin{array}{l}1.24 \\
\pm 0.02\end{array}$ & $\begin{array}{l}1.64 \\
\pm 0.02\end{array}$ & $\begin{array}{l}1.18 \\
\pm 0.03\end{array}$ & $\begin{array}{l}1.32 \\
\pm 0.02\end{array}$ & $\begin{array}{l}1.68 \\
\pm 0.03\end{array}$ & $\begin{array}{l}2.38 \\
\pm 0.04\end{array}$ & $\begin{array}{l}1.06 \\
\pm 0.03\end{array}$ & $\begin{array}{l}1.24 \\
\pm 0.01\end{array}$ & $\begin{array}{l}141.12 \\
\pm 0.02\end{array}$ & $\begin{array}{l}206.97 \\
\pm 0.02\end{array}$ & $\begin{array}{l}119.36 \\
\pm 0.02\end{array}$ & $\begin{array}{l}200.88 \\
\pm 0.09\end{array}$ & $\begin{array}{l}246.8 \\
\pm 0.16\end{array}$ & $\begin{array}{l}256.2 \\
\pm 0.24\end{array}$ & $\begin{array}{l}100.4 \\
\pm 0.41\end{array}$ & $\begin{array}{l}135.5 \\
\pm 0.21\end{array}$ \\
\hline & $\mathrm{C}$ & $\begin{array}{l}1.16 \\
\pm 0.03\end{array}$ & $\begin{array}{l}1.38 \\
\pm 0.02\end{array}$ & $\begin{array}{l}1.12 \\
\pm 0.03\end{array}$ & $\begin{array}{l}1.24 \\
\pm 0.02\end{array}$ & $\begin{array}{l}1.36 \\
\pm 0.02\end{array}$ & $\begin{array}{l}1.96 \\
\pm 0.02\end{array}$ & $\begin{array}{l}1.02 \\
\pm 0.004\end{array}$ & $\begin{array}{l}1.14 \\
\pm 0.02\end{array}$ & $\begin{array}{l}112.89 \\
\pm 0.05\end{array}$ & $\begin{array}{l}197.56 \\
\pm 0.03\end{array}$ & $\begin{array}{l}110.68 \\
\pm 0.06\end{array}$ & $\begin{array}{l}158.76 \\
\pm 0.18\end{array}$ & $\begin{array}{l}204.4 \\
\pm 0.48\end{array}$ & $\begin{array}{l}178.5 \\
\pm 0.42\end{array}$ & $\begin{array}{l}186.5 \\
\pm 0.20\end{array}$ & $\begin{array}{l}108.4 \\
\pm 0.36\end{array}$ \\
\hline \multirow[t]{3}{*}{$\begin{array}{l}\text { Borjan } \\
\text { Segment }\end{array}$} & A & $\begin{array}{l}1.29 \\
\pm 0.04\end{array}$ & $\begin{array}{l}1.58 \\
\pm 0.05\end{array}$ & $\begin{array}{l}1.24 \\
\pm 0.02\end{array}$ & $\begin{array}{l}1.36 \\
\pm 0.03\end{array}$ & $\begin{array}{l}1.48 \\
\pm 0.04\end{array}$ & $\begin{array}{l}2.08 \\
\pm 0.02\end{array}$ & $\begin{array}{l}1.15 \\
\pm 0.03\end{array}$ & $\begin{array}{l}1.29 \\
\pm 0.04\end{array}$ & $\begin{array}{l}238.3 \\
\pm 0.32\end{array}$ & $\begin{array}{l}263.42 \\
\pm 0.14\end{array}$ & $\begin{array}{l}216.83 \\
\pm 0.15\end{array}$ & $\begin{array}{l}254.86 \\
\pm 0.13\end{array}$ & $\begin{array}{l}251.5 \\
\pm 0.38\end{array}$ & $\begin{array}{l}266.4 \\
\pm 0.34\end{array}$ & $\begin{array}{l}135.8 \\
\pm 0.65\end{array}$ & $\begin{array}{l}174.6 \\
\pm 0.16\end{array}$ \\
\hline & B & $\begin{array}{l}1.22 \\
\pm 0.03\end{array}$ & $\begin{array}{l}1.35 \\
\pm 0.04\end{array}$ & $\begin{array}{l}1.17 \\
\pm 0.03\end{array}$ & $\begin{array}{l}1.24 \\
\pm 0.04\end{array}$ & $\begin{array}{l}1.36 \\
\pm 0.03\end{array}$ & $\begin{array}{l}1.86 \\
\pm 0.03\end{array}$ & $\begin{array}{l}1.08 \\
\pm 0.04\end{array}$ & $\begin{array}{l}1.22 \\
\pm 0.02\end{array}$ & $\begin{array}{l}213.25 \\
\pm 0.30\end{array}$ & $\begin{array}{l}225.79 \\
\pm 0.32\end{array}$ & $\begin{array}{l}208.35 \\
\pm 0.36\end{array}$ & $\begin{array}{l}237.65 \\
\pm 0.13\end{array}$ & $\begin{array}{l}238.6 \\
\pm 0.32\end{array}$ & $\begin{array}{l}248.2 \\
\pm 0.15\end{array}$ & $\begin{array}{l}116.5 \\
\pm 0.02\end{array}$ & $\begin{array}{l}129.7 \\
\pm 0.20\end{array}$ \\
\hline & $\mathrm{C}$ & $\begin{array}{l}1.14 \\
\pm 0.02\end{array}$ & $\begin{array}{l}1.21 \\
\pm 0.03\end{array}$ & $\begin{array}{l}1.08 \\
\pm 0.03\end{array}$ & $\begin{array}{l}1.16 \\
\pm 0.04\end{array}$ & $\begin{array}{l}1.19 \\
\pm 0.038\end{array}$ & $\begin{array}{l}1.61 \\
\pm 0.020\end{array}$ & $\begin{array}{l}1.02 \\
\pm 0.033\end{array}$ & $\begin{array}{l}1.08 \\
\pm 0.032\end{array}$ & $\begin{array}{l}200.7 \\
\pm 0.73\end{array}$ & $\begin{array}{l}219.52 \\
\pm 0.10\end{array}$ & $\begin{array}{l}195.45 \\
\pm 0.04\end{array}$ & $\begin{array}{l}208.46 \\
\pm 0.28\end{array}$ & $\begin{array}{l}224.5 \\
\pm 0.28\end{array}$ & $\begin{array}{l}241.4 \\
\pm 0.48\end{array}$ & $\begin{array}{l}108.5 \\
\pm 0.24\end{array}$ & $\begin{array}{l}113.8 \\
\pm 0.17\end{array}$ \\
\hline \multirow[t]{3}{*}{$\begin{array}{l}\text { Padumoni } \\
\text { Segment }\end{array}$} & A & $\begin{array}{l}1.06 \\
\pm 0.03\end{array}$ & $\begin{array}{l}1.13 \\
\pm 0.03\end{array}$ & $\begin{array}{l}1.02 \\
\pm 0.03\end{array}$ & $\begin{array}{l}1.24 \\
\pm 0.02\end{array}$ & $\begin{array}{l}1.42 \\
\pm 0.03\end{array}$ & $\begin{array}{l}1.76 \\
\pm 0.03\end{array}$ & $\begin{array}{l}0.96 \\
\pm 0.03\end{array}$ & $\begin{array}{l}1.11 \\
\pm 0.04\end{array}$ & $\begin{array}{l}106.62 \\
\pm 2.1\end{array}$ & $\begin{array}{l}200.7 \\
\pm 2.0\end{array}$ & $\begin{array}{l}101.62 \\
\pm 2.36\end{array}$ & $\begin{array}{l}196.55 \\
\pm 1.46\end{array}$ & $\begin{array}{l}259.0 \\
\pm 2.5\end{array}$ & $\begin{array}{l}268.2 \\
\pm 2.6\end{array}$ & $\begin{array}{l}101.6 \\
\pm 1.26\end{array}$ & $\begin{array}{r}187.5 \\
\pm 1.80\end{array}$ \\
\hline & B & $\begin{array}{l}1.02 \\
\pm 0.03\end{array}$ & $\begin{array}{l}1.09 \\
\pm 0.02\end{array}$ & $\begin{array}{l}0.98 \\
\pm 0.04\end{array}$ & $\begin{array}{l}1.12 \\
\pm 0.03\end{array}$ & $\begin{array}{l}1.34 \\
\pm 0.04\end{array}$ & $\begin{array}{l}1.58 \\
\pm 0.05\end{array}$ & $\begin{array}{l}0.92 \\
\pm 0.03\end{array}$ & $\begin{array}{l}1.06 \\
\pm 0.03\end{array}$ & $\begin{array}{l}103.48 \\
\pm 2.28\end{array}$ & $\begin{array}{l}169.34 \\
\pm 3.10\end{array}$ & $\begin{array}{l}100.78 \\
\pm 2.32\end{array}$ & $\begin{array}{l}174.72 \\
\pm 1.56\end{array}$ & $\begin{array}{l}241.8 \\
\pm 2.60\end{array}$ & $\begin{array}{l}241.6 \\
\pm 1.50\end{array}$ & $\begin{array}{r}101.5 \\
\pm 1.20\end{array}$ & $\begin{array}{r}158.4 \\
\pm 1.30\end{array}$ \\
\hline & $\mathrm{C}$ & $\begin{array}{l}0.91 \\
\pm 0.04\end{array}$ & $\begin{array}{l}1.05 \\
\pm 0.024\end{array}$ & $\begin{array}{l}0.85 \\
\pm 0.04\end{array}$ & $\begin{array}{l}1.08 \\
\pm 0.03\end{array}$ & $\begin{array}{l}1.18 \\
\pm 0.04\end{array}$ & $\begin{array}{l}1.23 \\
\pm 0.04\end{array}$ & $\begin{array}{l}0.82 \\
\pm 0.02\end{array}$ & $\begin{array}{l}1.05 \\
\pm 0.02\end{array}$ & $\begin{array}{l}100.35 \\
\pm 0.98\end{array}$ & $\begin{array}{l}131.71 \\
\pm 2.69\end{array}$ & $\begin{array}{l}100.08 \\
\pm 1.55\end{array}$ & $\begin{array}{l}126.64 \\
\pm 0.93\end{array}$ & $\begin{array}{l}232.2 \\
\pm 2.50\end{array}$ & $\begin{array}{l}234.6 \\
\pm 2.60\end{array}$ & $\begin{array}{l}98.6 \\
\pm 1.50\end{array}$ & $\begin{array}{r}142.7 \\
\pm 0.65 \\
\end{array}$ \\
\hline
\end{tabular}

$\mathrm{I}=$ infested, UI= un- infested, A: $0-15 \mathrm{~cm}, \quad \mathrm{~B}: 5-25 \mathrm{~cm}, \mathrm{C}: 25-50 \mathrm{~cm}$ 
Table.5 Available Phosphorus and Potassiun content of soil in Bherjan-Borjan-Padumoni WLS

\begin{tabular}{|c|c|c|c|c|c|c|c|c|c|c|c|c|c|c|c|c|c|}
\hline \multirow{3}{*}{ 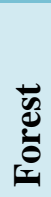 } & & \multicolumn{8}{|c|}{ Available Phosphorus (Kg ha $\left.{ }^{-1}\right)$} & \multicolumn{8}{|c|}{ Available Potassium (Kg ha $\left.{ }^{-1}\right)$} \\
\hline & & \multicolumn{2}{|c|}{ Pre-M } & \multicolumn{2}{|c|}{ Monsoon } & \multicolumn{2}{|c|}{ Post- M } & \multicolumn{2}{|c|}{ Winter } & \multicolumn{2}{|l|}{ Pre-M } & \multicolumn{2}{|c|}{ Monsoon } & \multicolumn{2}{|c|}{ Post- M } & \multicolumn{2}{|c|}{ Winter } \\
\hline & & $\mathbf{I N}$ & UI & IN & UI & IN & $\mathbf{U I}$ & IN & UI & IN & $\mathbf{U I}$ & IN & UI & IN & $\mathbf{U I}$ & IN & $\mathbf{U I}$ \\
\hline \multirow{6}{*}{ 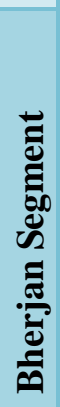 } & A & 28.98 & 31.89 & 28.64 & 30.58 & 16.35 & 25.63 & 15.86 & 23.76 & 254.72 & 288.44 & 188.99 & 236.67 & 214.62 & 258.1 & 157.5 & 205.8 \\
\hline & & \pm 0.02 & \pm 0.03 & \pm 0.05 & \pm 0.02 & \pm 0.01 & \pm 0.04 & \pm 0.06 & \pm 0.04 & \pm 0.06 & \pm 0.06 & \pm 0.77 & \pm 0.06 & \pm 0.02 & \pm 0.06 & \pm 0.44 & \pm 0.77 \\
\hline & B & 28.78 & 22.67 & 25.57 & 26.86 & 15.96 & 22.77 & 13.64 & 19.62 & 223.9 & 231.75 & 188.95 & 212.57 & 208.55 & 229.6 & 142.8 & 188.4 \\
\hline & & \pm 0.04 & \pm 0.03 & \pm 0.03 & \pm 0.03 & \pm 0.05 & \pm 0.02 & \pm 0.04 & \pm 0.02 & \pm 0.11 & \pm 0.64 & \pm 0.06 & \pm 0.06 & \pm 0.77 & \pm 0.28 & \pm 0.20 & \pm 0.69 \\
\hline & $\mathrm{C}$ & 26.56 & 19.98 & 21.68 & 24.76 & 14.77 & 20.85 & 12.49 & 15.44 & 169.87 & 196.59 & 174.6 & 192.66 & 158.4 & 208.5 & 128.4 & 164.5 \\
\hline & & \pm 0.03 & \pm 0.05 & \pm 0.03 & \pm 0.03 & \pm 0.04 & \pm 0.02 & \pm 0.04 & \pm 0.04 & \pm 0.09 & \pm 0.57 & \pm 0.16 & \pm 0.02 & \pm 0.36 & \pm 0.41 & \pm 0.41 & \pm 0.36 \\
\hline \multirow{6}{*}{ 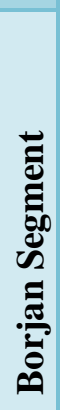 } & A & 34.44 & 36.67 & 27.85 & 29.88 & 15.28 & 27.25 & 12.89 & 24.56 & 234.72 & 259.44 & 208.44 & 224.58 & 164.4 & 173.2 & 138.5 & 156.8 \\
\hline & & \pm 0.04 & \pm 0.06 & \pm 0.04 & \pm 0.05 & \pm 0.02 & \pm 0.05 & \pm 0.03 & \pm 0.04 & \pm 0.06 & \pm 0.06 & \pm 0.04 & \pm 0.14 & \pm 0.02 & \pm 0.32 & \pm 0.32 & \pm 0.16 \\
\hline & B & 29.30 & 26.77 & 26.75 & 24.56 & 14.66 & 25.66 & 10.48 & 18.54 & 218.50 & 227.75 & 195.80 & 208.43 & 152.8 & 164.8 & 124.7 & 144.5 \\
\hline & & \pm 0.04 & \pm 0.03 & \pm 0.03 & & \pm 0.02 & \pm 0.04 & \pm 0.04 & \pm 0.06 & \pm 0.20 & \pm 0.13 & \pm 0.03 & \pm 0.20 & \pm 0.16 & \pm 0.16 & \pm 0.20 & \pm 0.57 \\
\hline & $\mathrm{C}$ & 22.34 & 20.11 & 18.68 & 20.69 & 13.59 & 24.72 & 10.08 & 12.52 & 156.87 & 185.54 & 185.25 & 189.57 & 136.4 & 156.8 & 118.4 & 132.5 \\
\hline & & \pm 0.05 & \pm 0.07 & \pm 0.07 & \pm 0.04 & \pm 0.07 & \pm 0.05 & \pm 0.03 & \pm 0.06 & \pm 0.06 & \pm 0.04 & \pm 0.03 & \pm 0.80 & \pm 0.20 & \pm 0.16 & \pm 0.40 & \pm 0.24 \\
\hline \multirow{6}{*}{ 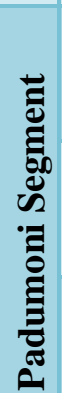 } & A & 28.55 & 28.59 & 27.58 & 26.67 & 21.87 & 26.64 & 12.55 & 14.69 & 248.79 & 268.44 & 207.64 & 237.3 & 213.55 & 254.6 & 132.6 & 168.6 \\
\hline & & \pm 0.04 & \pm 0.06 & \pm 0.05 & \pm 0.06 & \pm 0.07 & \pm 0.06 & \pm 0.07 & \pm 0.04 & \pm 1.69 & \pm 0.06 & \pm 0.05 & \pm 0.05 & \pm 0.10 & \pm 0.23 & \pm 0.25 & \pm 0.33 \\
\hline & B & 24.56 & 22.64 & 22.49 & 23.88 & 21.56 & 26.46 & 10.24 & 13.46 & 211.14 & 239.98 & 190.85 & 196.54 & 228.5 & 225.8 & 118.8 & 149.8 \\
\hline & & \pm 0.05 & \pm 0.04 & \pm 0.06 & \pm 0.05 & \pm 0.09 & \pm 0.04 & \pm 0.03 & \pm 0.04 & \pm 0.08 & \pm 0.03 & \pm 0.09 & \pm 0.04 & \pm 0.19 & \pm 0.72 & \pm 0.82 & \pm 0.40 \\
\hline & $\mathrm{C}$ & 19.89 & 16.99 & 18.47 & 22.55 & 20.78 & 23.77 & 9.76 & 10.54 & 153.23 & 178.49 & 148.48 & 158.65 & 140.6 & 168.6 & 108.6 & 134.4 \\
\hline & & \pm 0.04 & \pm 0.04 & \pm 0.04 & \pm 0.06 & \pm 0.02 & \pm 0.04 & \pm 0.05 & \pm 0.05 & \pm 0.24 & \pm 0.11 & \pm 0.11 & \pm 0.07 & \pm 0.24 & \pm 0.28 & \pm 0.24 & \pm 0.56 \\
\hline
\end{tabular}

$\mathrm{I}=$ infested, $\mathrm{UI}=$ un- infested, A: 0-15 cm, B: $5-25 \mathrm{~cm}, \mathrm{C}: 25-50 \mathrm{~cm}$ 
Table.6 Correlation of different parameters relevant to Mikania infestation A: Bherjan B: Borjan $\mathrm{C}$ : Podumoni

\begin{tabular}{|l|l|l|l|l|}
\hline Season & ST & LI & BM & SM \\
\hline \multicolumn{5}{|c|}{ Bherjan } \\
\hline ST & - & 0.7262 & 0.5362 & 0.4248 \\
\hline LI & 0.7262 & - & $0.9471^{* *}$ & 0.4905 \\
\hline BM & 0.5362 & $\mathbf{0 . 9 4 7 1}^{* *}$ & - & 0.6443 \\
\hline SM & 0.4248 & 0.4905 & 0.6443 & - \\
\hline \multicolumn{5}{|c|}{ Borjan } \\
\hline ST & - & 0.8102 & 0.8275 & 0.2908 \\
\hline LI & 0.8102 & - & $0.9772^{* *}$ & 0.2678 \\
\hline BM & 0.8275 & $\mathbf{0 . 9 7 7 2}$ & - & 0.0751 \\
\hline SM & 0.2908 & 0.2678 & 0.0751 & - \\
\hline \multicolumn{5}{|c|}{ Padumoni } \\
\hline ST & - & 0.2855 & 0.6466 & 0.4166 \\
\hline LI & 0.2855 & - & $0.9156^{* *}$ & 0.6438 \\
\hline BM & 0.6466 & $\mathbf{0 . 9 1 5 6}$ & - & $0.8966^{*}$ \\
\hline SM & 0.4166 & 0.6438 & $\mathbf{0 . 8 6 6 6}^{*}$ & - \\
\hline
\end{tabular}

ST: soil temperature LI: light interception BM: biomass SM: soil moisture

Fig.1 Map of Bherjan-Borjan-Padumoni WLS

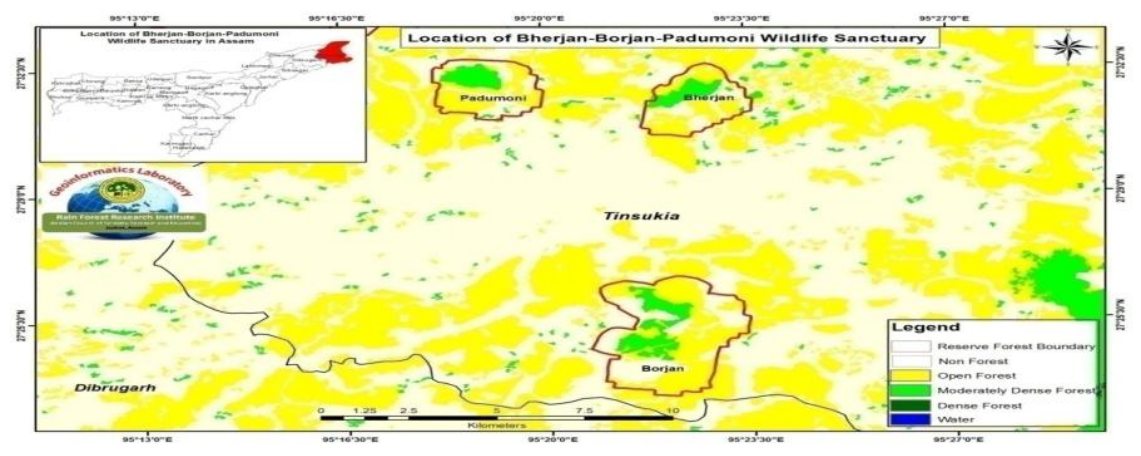

Fig.2 Structural changes of Mikania micrantha in different season

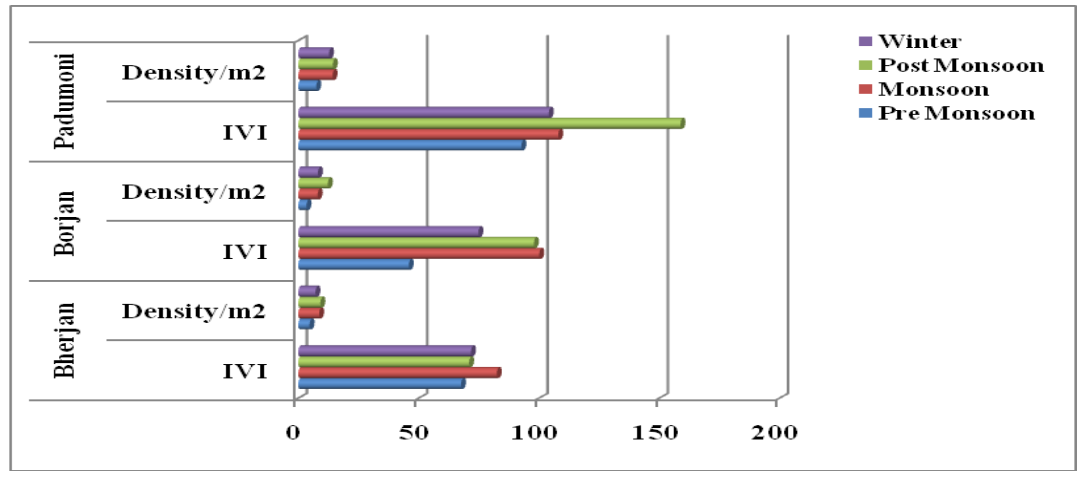


Fig.3 Comparative assessment of light interception \% and Mikania biomass at different growth season in Bherjan-Borjan-Padumoni WLS

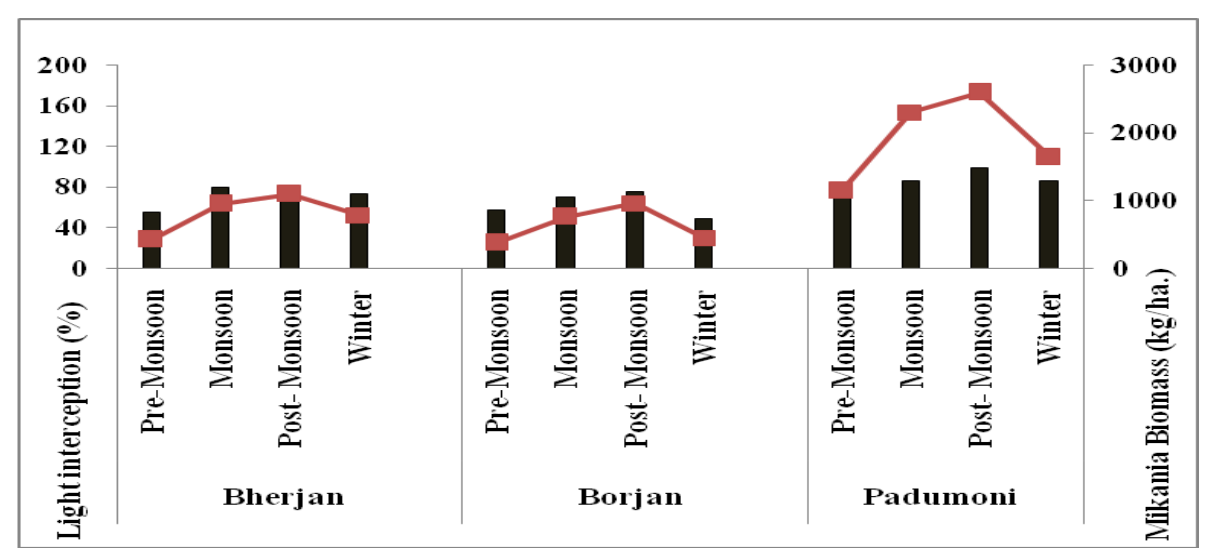

Plate.1 Glimpses of Bherjan- Borajan-Podumoni Wild Life Sanctuary A\& B: Fragmentation by road and Infestation of Mikania in Bherjan segment $\mathrm{C}$ : Amomum maximum- an important spice species in forest floor of Borjan segment D: Mikania Converted Padumoni segment -a degraded forest
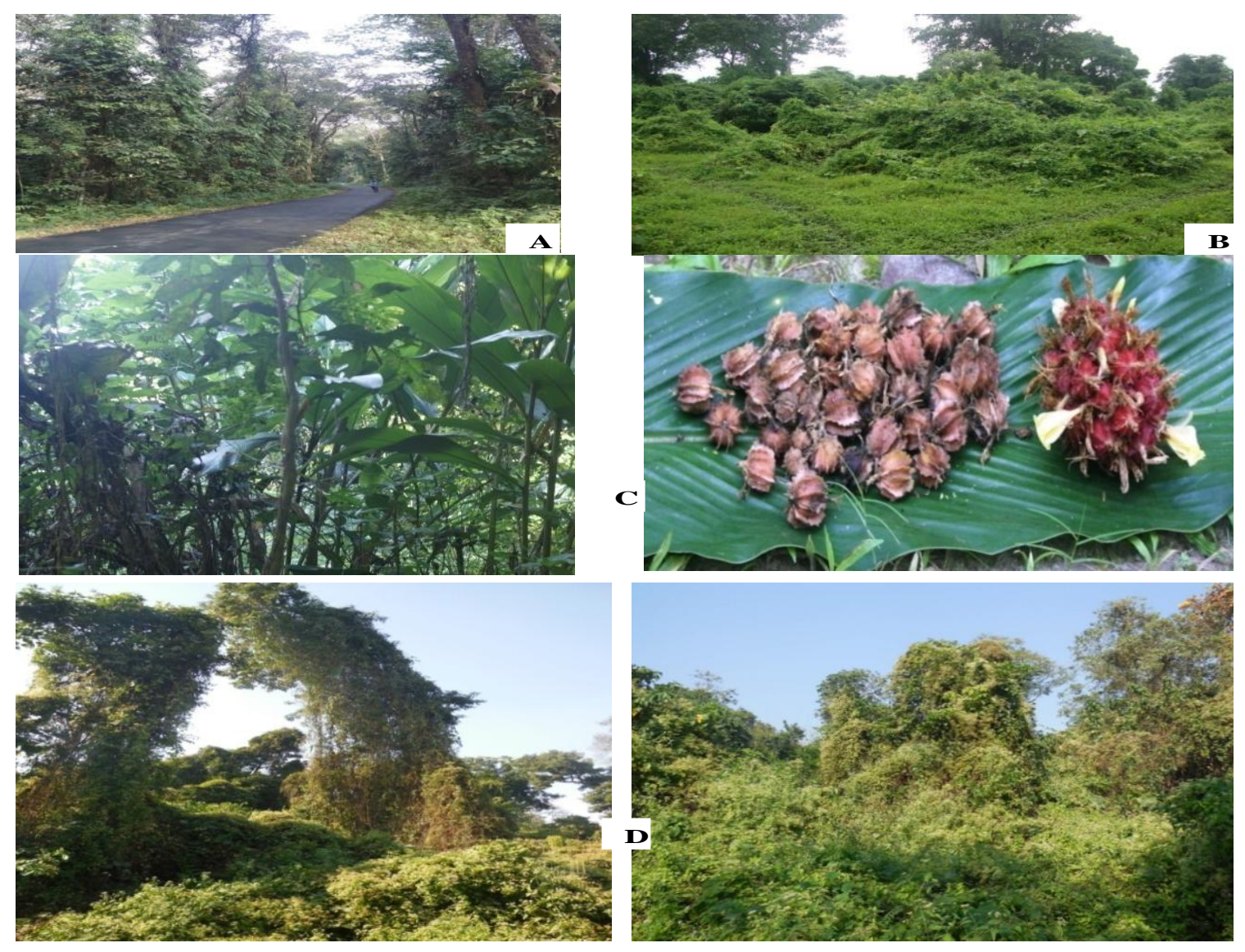
Vegetation played a major role in soil organic carbon content which increases through ample supply of organic residues by litter decomposition and mineralization. The value of organic carbon was noticed high in post monsoon season of Bherjan segment followed by Borjan and Padumoni segment. Significant increment of organic carbon was recorded in top layers due to accumulation of sizeable amount of humus and in subsurface layers it consisted of leached fraction and decayed products of underground biomass which decreased with soil depth (Sharma and Gupta, 2015). During present study higher values of organic $\mathrm{C}$ and $\mathrm{N}$ were observed in un-infested forest compared to Mikania invaded areas (Table 4). The invasive plants have potential to alter the $\mathrm{N}$ cycling process through their litter production (Allison and Vitousek, 2004). Chen et al., (2009) reported that invasion of $M$. micrantha decreased nitrogen content in soil due to quick absorption. They also observed more total $\mathrm{C}$ and $\mathrm{N}$ in soil from the rhizosphere of Mikania.

Maximum litter accumulation by the invasive species and nutrient release from decomposing litter possibly created condition for continuous invasion, Kourtev et al., (2003). Nitrogen content was found maximum in post monsoon season of all the study sites and in winter season the value was recorded least with the increase of soil depth. Value of phosphorus ranged from $14.69 \mathrm{~kg}$ /ha to $36.67 \mathrm{~kg} / \mathrm{ha}$ in surface layer of different un infested forests sites, however it was ranged from $12.55 \mathrm{~kg} / \mathrm{ha}$ to $34.44 \mathrm{~kg} / \mathrm{ha}$ in infested sites. In Mikania infested forest potassium concentration was less in compared to un infested forest sites which was also supported by the observation of Gogoi et al., (2011). Highest value of phosphorus and potassium were observed in pre monsoon season and least value was in winter season (Table 5).
Mikania has an optimum growth stage in post monsoon season along with less environmental variation. Therefore, this season was the ideal for interpretation of correlation on microenvironment data. Correlation of environmental data, soil data and the biomass data recorded from Mikania infested forest sites revealed that Mikania biomass was having highly significant correlation with the interception of light by the canopy among all the segment of BherjanBorjan-Padumoni WLS. Borjan and Bherjan segment showed positive correlation with soil temperature and light interception, where as in Padumoni segment biomass and soil moisture percent exhibited significant correlation that indicated the maximum spreading out of Mikania (Table 6).

Impact of changes of micro environmental factors on plants is more acute in forest ecosystems, particularly in rain forests, where stratification is governed by a number of environmental factors. Prevalence of such factors of environmental change for a prolonged period may be responsible for permanent shift of vegetation composition, soil seed bank as well as the corresponding ecological functions. Randall (1996) stated that alien invasive species were the second largest threat to biodiversity. M. micrantha is one of the most troublesome driver species of Assam and extreme capacity for alteration of native community and entire ecosystem functions.

It showed tremendous capability to form monoculture community that was supported by Mack et al., (2000). Smothering effect of this fast growing vine triggered the deciduous species and displacing several indigenous species which may alter the forest scenario in near future. Maintaining close canopy coverage of the forest might be one of the precautionary measures against the rapid invasion of the weed. 


\section{Acknowledgement}

Authors would like to express deep sense of gratitude and sincere thanks to the Director, Rain Forest Research Institute, Jorhat whose advice and encouragement were vital. Authors also thankful to the State Forest Department of Assam, Divisional Forest Officer Tinsukia and their staff for help and permission to do work in the Wild Life Sanctuary.

\section{References}

Acharya, R. and Shrestha, B.B. 2012. Physicochemical characteristics of soil of a mixed Shorea robusta forest Rupandehi district, Nepal. J. Nat. Hist. Mus. 26: 155-162.

Allison, S.D., Vitousek, P.M.,2004. Rapid nutrient cycling in leaf litter from invasive plants in Hawaii. Ecologia $141 ; 612-619$

Anonymous, 2007. Ill effect of parthenium on human and animal health. AICRP on weed control, Annual Report, 160-165.

Anup, A., Bhandari, G., Wagle, S.P. and Banjade, Y. 2013. Status of soil fertility in a community forest of Nepal. Int. J. Env. 1(1): 56-67.

Aravind, N.A., Rao, D., Ganeshaiah, K.N., Uma Shankar, R. and Poulsen, J.G. 2010. Impact of invasive plant Lantana camara, on birds assemblages at Male Mahadeshwara Reserve Forest, South India. Trop Ecol. 51:325-338.

Barua, Kuntala N. and Hazarika, P.2016 Tree diversity and regeneration status in Dilli Forest Reserve of Assam following weed invasion J. Eco. Tax. Bot., 40(3\&4): 89-98.

Brown, J.R., J.C. Scalan and J.G. Mcivor. 1998. Competition by herbs as a limiting factor in shrub invasion in grassland. A test with different growth form. J. Veg. Sci., 9: 829-836.
Chen, B.M., Peng, S.L. and Ni, G.Y. 2009. Effects of the invasive plant Mikania micrantha H.B.K. on soil nitrogen availability through allelopathy in South China. Biol Invasions 11:12911299.

Drake, J.A., FDiCastri., R.H. Groover, F.J. Druger, H.A. Mooney, M. Rejmanek and Williams, M. (eds) 1989. Biological invasion: A Global perspective Scope 37; John Wiley and Sons Ltd., Chichester, UK.

Ehrenfeld, J.G. 2003. Effect of exotic plant invasions on soil nutrient cycling processes. Ecosystems, 6, 503- 523.

Feng, Y.L.2007. Photosynthesis, nitrogen allocation and specific leaf area in invasive Eupatorium adenophorum and native Eupatorium japonicum grown at different irradiances. Physiologia Plantarum 133: 318-326.

Huang, Z.L., Cao, H.L., Liang, X.D., Ye, W.H., Feng, H.L. and Cai, C.X. 2000. The growth and damaging effect of Mikania micrantha in different habitats. J. Trop Subtrop Bot. 8:131138.

Jackson, M.L. 1973. Soil Chemical Analysis. Prantice Hall of India.

Jha M.N., Pande Padmankar and Pathak, T.C.1979. Studies on the changes in the physico-chemical properties of Tripura Soils as a result of Jhuming. Ind. For. 105(6): 436-443.

Jha, K.K. 2005. Storage and flux of organic carbon in young Tectonagrandis plantation in moist deciduous forest. Ind. For. 131(5): 647-659.

Kershaw, K.K. 1973. Quantitative and dynamic Plant Ecology Edward Arnold London 308 pp.

Lee, K.A. and Klasing, K.C. 2004. A role for immunology in invasion biology. Trends Ecol. Evol.19, 523-529

Lowe, S., Browne, M., Boudjelas, S. and DE Poorter, M. 2000. 100 of the World's 
Worst Invaisve Alien Species, A selection from the Global Invasive species database, Invasive Species Specialist Group (ISSG) Auckland, New Zealand. https://www.issg.org/booklet pdf (Accessed on 12 July, 2008).

Mack, R.N., Simberloff, D., Mark Lonsdale, W.,Evans, H., Clout, M. and Bazzaz, F. A. 2000. Biotic invasions: Causes, epidemiology, global consequences, and control. Ecol. Appl. 10, 689-710.

Pimm, S.L. 1986. Community structure and Stability. In: M.Soule (eds.) Conservation Biology: The science of scarcity and biodiversity. Sinuer, Sunderland, Massachusetts, USA. Pp: 309-329.

Mishra, R. 1968. Ecology Work Book. Oxford and IBH New Delhi, $244 \mathrm{pp}$.

Puzari, K.C., Bhuyan, R. P., Pranab, D. and Nath, H.K.D. 2010. Distribution of Mikania and its economic impact on tea ecosystem of Assam. Ind. J. For. 33(1): 71-76

Ram, A.K. 2008. Impact of Mikania micrantha on Rhinoceros habitat in Chitwan National Park, Chitwan Nepal. Thesis submitted for the Degree of Bachelor of Science in Forestry.

Randall, J.M.1996. Weed control for the preservation of Biological Diversity. Weed Technol, 10: 370-383.

Sankaran, K.V, Muraleedharan, P.K. and Anitha, V.2001. Integrated management of the alien invasive weed Mikania micrantha in the Western Ghats. KFRI Research Report No22:149

Singh, S.P. 2001. Biological control of alien invasive weeds in India. In: Sankaran K.V., Murthy, S.T. and Evans, H.C. (eds)., Alien Weeds in Moist Tropical Zones: Banes and Benefits. KFRI, India and CABI Bioscience, U.K. 172P, 2-4 Nov. 1999, pp. 11-19.

Swamy, P.S. and Ramakrishanan, P.S. 1987a. Effect of fire on population dynamics of Mikania micrantha H.B.K. during early succession after slash and burn agriculture (jhum) in North-East India. Weed Research 27: 397-403.

Swamy, P.S. and Ramakrishnan P.S. 1987b. Contribution of Mikania micrantha H.B.K., during secondary succession following slash and burn agriculture (Jhum) in northeast India. I. Biomass, litterfall and productivity. For. Ecol. Manage. 22: 239-249.

Tiwari, S., SiwakotI, M., Adhikari, B. \&Subedi, K. 2005. An inventory and assessment of invasive alien plant species of Nepal. 144pp.

Vasu, N.K. 2003. Management of invasive species at Kaziranga National Park. National seminar on Alien Invasive weeds in India. 27-29 $9^{\text {th }}$ April. Assam Agricultural University, Jorhat Assam. pp10.

\section{How to cite this article:}

Kuntala Neog Barua, Indrani P. Bora and Arundhati Baruah. 2020. Impact of Mikania micrantha Kunth ex HBK Invasion on Micro-environment Component of Bherjan- BorajanPodumoni Wild Life Sanctuary, Assam. Int.J.Curr.Microbiol.App.Sci. 9(09): 2900-2912. doi: https://doi.org/10.20546/ijcmas.2020.909.357 